\title{
Risk factors for preterm delivery among early onset cancer survivors: A Finnish register-based study
}

\section{Melin, Johanna}

2019-04-15

Melin , J , Heinävaara , S , Malila , N , Tiitinen , A , Gissler , M \& Madanat-Harjuoja , L 2019, ' Risk factors for preterm delivery among early onset cancer survivors: A Finnish register-based study ' , International Journal of Cancer , vol. 144 , no. 8 , pp. 1954-1961 . https://doi.org/10.1002/ijc.3

http://hdl.handle.net/10138/312555

https://doi.org/10.1002/ijc.31938

unspecified

publishedVersion

Downloaded from Helda, University of Helsinki institutional repository.

This is an electronic reprint of the original article.

This reprint may differ from the original in pagination and typographic detail.

Please cite the original version. 


\title{
Risk factors for preterm delivery among early onset cancer survivors: A Finnish register-based study
}

\author{
Johanna Melin (10) ${ }^{1,2}$, Sirpa Heinävaara ${ }^{1,3}$, Nea Malila ${ }^{1,4}$, Aila Tiitinen ${ }^{5}$, Mika Gissler $^{6,7}$ and Laura Madanat-Harjuoja ${ }^{1,8}$ \\ ${ }^{1}$ Finnish Cancer Registry, Institute for Statistical and Epidemiological Cancer Research, Helsinki, Finland \\ ${ }^{2}$ Department of Obstetrics and Gynecology, Kymenlaakso Central Hospital, Kotka, Finland \\ ${ }^{3}$ Department of Public Health, University of Helsinki, Helsinki, Finland \\ ${ }^{4}$ University of Tampere, Faculty of Social Sciences, Tampere, Finland \\ ${ }^{5}$ Department of Obstetrics and Gynecology, University of Helsinki, Helsinki University Hospital, Helsinki, Finland \\ ${ }^{6}$ National Institute for Health and Welfare, Information Services Department, Helsinki, Finland \\ ${ }^{7}$ Karolinska Institute, Department of Neurobiology, Care Science and Society, Stockholm, Sweden \\ ${ }^{8}$ Department of Pediatrics, University of Helsinki and Helsinki University Hospital, Helsinki, Finland
}

Previous studies have shown an elevated risk for preterm delivery among early onset cancer survivors. Whether the preterm delivery starts spontaneously, due to possible uterine damage because of cancer treatment, or is induced due to maternal conditions is unclear. Our aim was to assess pregnancy related conditions in female cancer survivors possibly underlying the elevated risk for preterm labor. Nationwide cancer and birth registries were merged to identify 1,753 first deliveries of cancer survivors (diagnosed below 40 years of age) and 5,123 first deliveries of matched female comparison subjects between January 1991 and December 2013. Conditional logistic regression models were used to estimate the risk for pregnancy related conditions adjusting for maternal age, gestational age and smoking. We found an overall increased risk for hospitalization during pregnancy (OR 1.45, 95\% Cl 1.25-1.68), intrahepatic cholestasis (OR 2.86, 95\% Cl 1.09-7.49), fear of childbirth (OR 2.25, 95\% Cl 1.31-3.85) and mental disorders and diseases of the nervous system complicating pregnancy and labor (OR 5.89, 95\% Cl 2.31-15.00). Among survivors, 129 (7.4\%) delivered preterm compared to 268 (5.2\%) comparisons subjects $(p=0.004)$. We found a statistically significant increased risk for preterm delivery among cancer survivors with vaginal bleeding (OR 1.35, 95\% Cl 1.07-1.71) and pre-eclampsia (1.35, 95\% Cl 1.06-1.72) compared to comparison subjects with the same condition. Health professionals treating these women should be aware of these risks. In general, however, our results are reassuring when it comes to pregnancies among cancer survivors.

\section{Introduction}

Long-term survival rates for most types of childhood cancers have improved markedly over the past decades. In a recent study, the 5-year survival rate for Finnish children diagnosed

Key words: female cancer survivors, pregnancy, preterm birth Abbreviations: FCR: Finnish Cancer Register; CPR: Central Population Register; MBR: Medical Birth Register; OR: Odd ratio; CI: Confidence Interval; ICCC3: International Classification of Childhood Cancer

Additional Supporting Information may be found in the online version of this article.

Conflicts of interest: No conflicts of interest exist.

Grant sponsor: Signe ja Ane Gyllenbergin Säätiö; Grant sponsor: Gyllenberg Foundation; Grant sponsor: Cancer Society of Finland; Grant numbers: 130079

DOI: $10.1002 / \mathrm{ijc} .31938$

History: Received 5 Jun 2018; Accepted 4 Oct 2018;

Online 23 Oct 2018

Correspondence to: Johanna Melin, Finnish Cancer Registry, Unioninkatu 22, 00130 Helsinki, Finland, E-mail: johanna. melin@cancer.fi; Tel.: +358505382526 in $2000-2010$ was as high as $81 \%,{ }^{1}$ paralleling European figures. $^{2}$ However, anticancer treatments can result in late complications up to decades after treatments. These adverse health conditions include impaired fertility ${ }^{3}$ as well as cardiovascular conditions ${ }^{4}$ and metabolic syndrome, ${ }^{5}$ among others.

Studies have shown an elevated risk for preterm delivery $^{6-13}$ among cancer survivors. Various explanations for this elevated risk have been offered. Abdomino-pelvic irradiation and chemotherapy have been identified as risk factors for preterm delivery and low birth weight of the fetus. ${ }^{9-11}$ In these survivors, the cancer treatment could lead to impaired uterine growth and reduced blood flow, resulting in uterine fibrosis and cervical shortening. This, in turn, could lead to premature contractions and rupture of the amniotic membranes, possibly resulting in spontaneous preterm delivery.

One alternate hypothesis is that the history of cancer potentially influences medical decision making placing the survivor under increased surveillance and lowering the threshold for medical interventions. ${ }^{14}$ Yet another explanation could be maternal pregnancy related conditions, which are known to necessitate medically induced preterm birth, ${ }^{15}$ the most 
What's new?

While previous studies suggest that the risk of preterm delivery is elevated among early-onset cancer survivors, the reasons underlying the association are unclear. In the present study, female cancer survivors diagnosed under age 40 in Finland were found to have an increased risk of hospitalization during pregnancy when compared to healthy subjects. Female early-onset cancer survivors also were at increased risk of fear of childbirth and mental conditions and disorders of the nervous system that potentially complicated pregnancy and labor. Risk of preterm delivery was further elevated among cancer survivors with vaginal bleeding and pre-eclampsia during pregnancy.

common ones being pre-eclampsia, gestational diabetes and placental pathologies. Previous studies have shown that pregnancy related hypertension, induction of labor and elective cesarean sections ${ }^{6,11,16}$ are more common among survivors.

The aim of our study was to explore pregnancy related conditions in female cancer survivors (diagnosed with cancer under the age of 40) possibly underlying the elevated risk for preterm labor.

\section{Materials and Methods}

Each Finnish citizen and permanent resident living in Finland is given a personal identity number which enables linkage between different registries and databases. This retrospective, nationwide registry based study used data derived from the linkage of three population-based registers.

\section{Registers}

The Finnish Cancer Registry (FCR) began registration in 1953 and coverage in this population-based registry is reported to be as high as $96 \%$ for solid tumors and $86 \%$ for hematological malignancies. Of all cancers, 93\% were morphologically verified. ${ }^{17}$ The registry data include details of primary cancer including anatomical site, histology, extent of disease, primary treatment (radiotherapy, chemotherapy and surgery) and time of diagnosis (month and year). Cancer cases are divided by tumor behavior at diagnosis into four different categories, namely benign, in situ carcinomas, semi-malignant and malignant tumors. In our cohort, in situ and basal cell carcinomas are excluded but we included benign and semi-malignant CNS tumors, as their treatment is similar to that of malignant brain tumors. Childhood cancer diagnoses are categorized using the International Classification of Childhood Cancer (ICCC3). ${ }^{18}$

The Central Population Register (CPR), founded in 1969, covers all Finnish residents and includes data on residential history and emigration, as well as possible date of death.

The Medical Birth Register (MBR) contains detailed data on all mothers giving birth and their children born in Finland from 1987 onwards. All live births and stillbirths with a birth weight of more than $500 \mathrm{~g}$ or a pregnancy continuing for a minimum of 22 weeks are recorded in this registry. Data of less than $0.1 \%$ of infants are missing in the MBR. ${ }^{19}$

Data on pregnancy related conditions requiring hospitalization and the indication for hospitalization have been recorded in the MBR from October 1990 onwards. Data on premature rupture of the amniotic membranes, gestational diabetes, fear of childbirth, mental disorders and diseases of the nervous system complicating pregnancy and childbirth are available since January 2004 in the MBR.

\section{Study design}

We identified 25,816 female cancer patients (hereafter referred to as survivors) diagnosed with a malignant neoplasm or benign brain tumor up to the age of 40 years between January 1957 and December 2012 from the FCR (Fig. 1). From the CPR, we sampled five age-matched female comparison subjects per case $(129,080)$. After excluding comparisons that were diagnosed with early onset cancer (7,726 women), 121,353 population comparisons remained.

By further linkage to the MBR, we identified 8,097 survivors and 45,953 comparison subjects with deliveries between January 1991 and December 2013.

After excluding women whose cancer was diagnosed during pregnancy or who had given birth before cancer diagnosis, 3,005 survivors were left.

To eliminate possible influences of obstetric history on the endpoint studied, only singleton, first deliveries were included, leaving us with 1,952 survivors. Not all survivors had matched, female comparison subjects with first deliveries, which is why only 1,753 survivors and their 5,123 matched comparisons were included in the study (Fig. 1).

The primary, pregnancy related outcomes, analyzed were: hospital stays exceeding 7 days around delivery and hospitalization during pregnancy (due to any cause and further sub classified into threatened preterm labor, unexplained vaginal bleeding, pre-eclampsia and other reasons than vaginal bleeding, pre-eclampsia or threatened preterm labor). Threatened preterm labor was defined as hospitalization due to cervical shortening or premature contractions and pre-eclampsia was defined as gestational hypertension and/or pre-eclampsia including essential hypertension diagnosed before pregnancy. Less severe conditions of threatened preterm labor, vaginal bleeding, pre-eclampsia and other conditions that did not require hospitalization were excluded. Gestational diabetes was defined as a pathological 2 -h oral glucose tolerance test result. ICD-10 diagnostic codes were used to identify the after conditions from the MBR data: premature rupture of the membranes (O42.0, O42.1, O42.2 and O42.9), intrahepatic cholestasis (O26.6) fear of childbirth (O99.80), mental disorders and diseases of the nervous system complicating pregnancy and childbirth (O99.3 which include mental and 


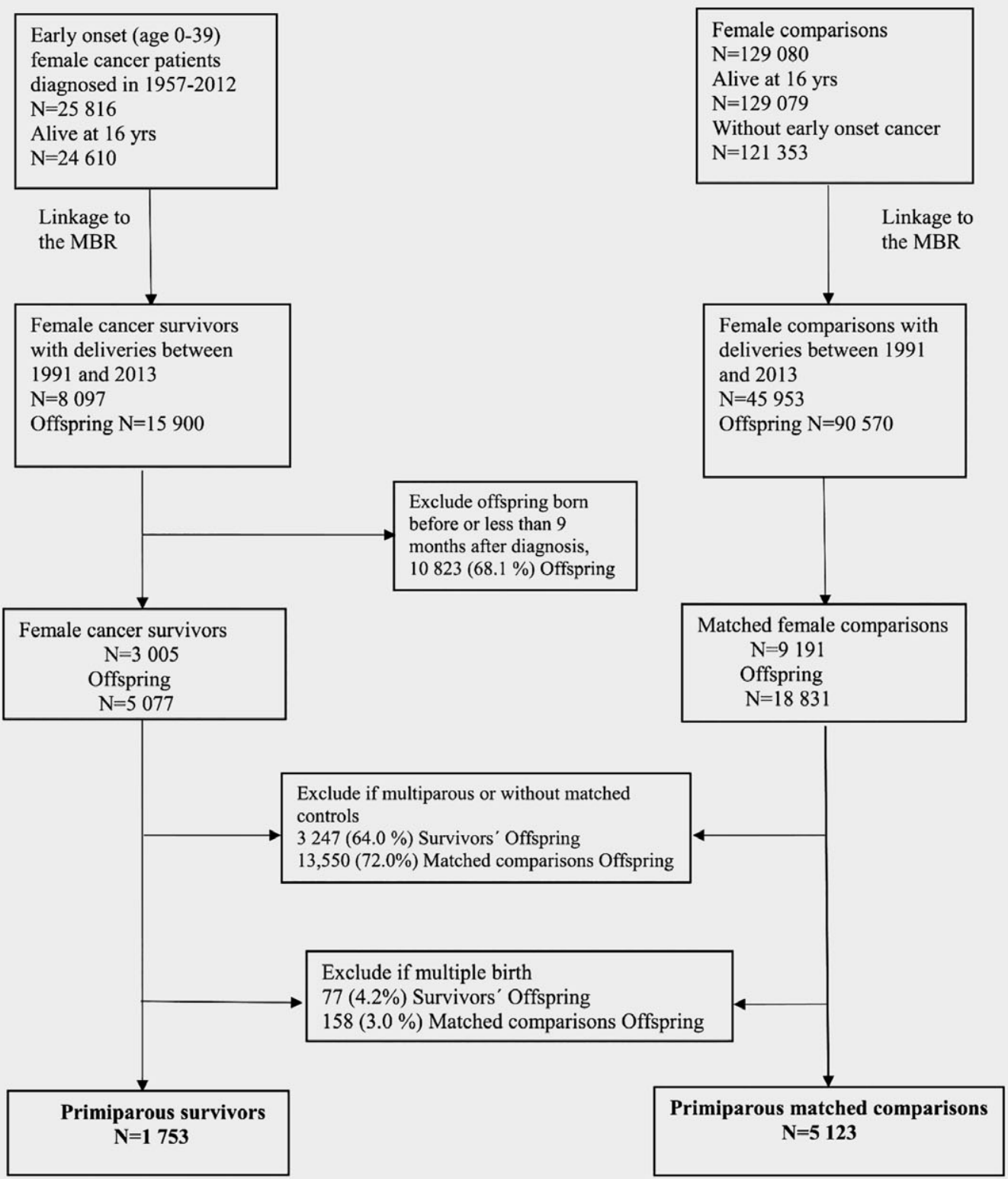

Figure 1. Female cancer survivors and their matched (by gender and age) female comparison group. Criteria for inclusion of deliveries and number of mothers and offspring included in the final analysis.

behavioral disorders F00-F99 and diseases of the nervous system G00-G99 in ICD-10). According to Finnish clinical guidelines, pregnant women are asked about their feelings and attitudes toward childbirth in primary health care. Those who are particularly fearful are referred to specialist maternity care. Normal worries and minor fear of childbirth are treated in primary health care. Finland has introduced a specific ICD-10 code for fear of childbirth (O99.80), which we utilized to identify these women.

\section{Statistical analysis}

Differences between survivors and comparison subjects in categorical variables were analyzed using the $\chi^{2}$-test. Conditional logistic regression analysis was used to estimate odd ratios (ORs) with 95\% confidence intervals (CI) for obstetric complications categorized as dichotomous variables comparing survivors to the comparison group, adjusting for maternal age at delivery, gestational age and maternal smoking. The level of statistical significance was set at $p<0.05$. 
Table 1. Descriptive characteristics of postdiagnosis first pregnancies of female cancer survivors and first pregnancies of a female comparison group

\begin{tabular}{|c|c|c|c|c|}
\hline Characteristics & Subcategory & Survivors $(N=1,753) N(\%)$ & Comparisons $(N=5,123) N(\%)$ & $p$ value \\
\hline \multirow[t]{4}{*}{ Age at delivery } & $<25$ & $331(18.88)$ & $1,515(29.57)$ & $<0.001$ \\
\hline & $25-29$ & $599(34.17)$ & $2,111(41.21)$ & \\
\hline & $30-34$ & $552(31.49)$ & $1,149(22.43)$ & \\
\hline & 35 or more & $271(15.46)$ & $348(6.79)$ & \\
\hline \multirow[t]{2}{*}{ Time period of delivery } & $1991-2002$ & $665(37.93)$ & $2,585(50.46)$ & $<0.001$ \\
\hline & $2003-2013$ & $1,088(62.07)$ & $2,538(49.54)$ & \\
\hline \multirow[t]{3}{*}{ Maternal smoking } & No & $1,494(85.23)$ & $4,130(80.62)$ & 0.056 \\
\hline & Yes & $227(12.95)$ & $885(17.28)$ & \\
\hline & Missing & $32(1.83)$ & $108(2.11)$ & \\
\hline \multirow[t]{2}{*}{ Infant sex } & Male & $911(51.97)$ & $2,643(51.59)$ & 0.785 \\
\hline & Female & $842(48.03)$ & $2,480(48.41)$ & \\
\hline \multirow[t]{5}{*}{ Gestational age } & $\ll 32$ & $32(1.83)$ & $42(0.82)$ & 0.004 \\
\hline & $32-36$ & $97(5.53)$ & $226(4.41)$ & \\
\hline & $37-41$ & $1,513(86.31)$ & 4,528 (88.39) & \\
\hline & 42 or more & $109(6.22)$ & $316(6.17)$ & \\
\hline & Missing & $2(0.11)$ & $11(0.21)$ & \\
\hline \multirow[t]{5}{*}{ Birth weight (g) } & $\ll 1,500$ & $31(1.77)$ & $36(0.70)$ & 0.002 \\
\hline & $1,500-2,499$ & $73(4.16)$ & $163(3.18)$ & \\
\hline & $2,500-3,999$ & $1,421(81.06)$ & $4,218(82.33)$ & \\
\hline & $4,000-4,499$ & 205 (11.69) & $619(12.08)$ & \\
\hline & 4,500 or more & $23(1.31)$ & $87(1.70)$ & \\
\hline
\end{tabular}

To identify possible patient and/or cancer treatment related risk factors, we performed sub-analyses on all above mentioned pregnancy related outcomes (except intrahepatic cholestasis and mental disorders and diseases of the nervous system which were excluded due to small numbers), stratifying by time from diagnosis to delivery ( $0-5$ years, 6-10 years and over 10 years), cancer treatment (chemotherapy, radiotherapy and surgery only) and diagnostic age. Pediatric patients were defined as survivors treated at the age of 0-14 years, adolescents and young adults at the age of 15-24 years and adults at the age of 25-39 years. In these analyses, 95\% CIs were corrected for multiple comparisons using the Bonferroni correction. Supplementary analyses were performed by further analyzing survivors treated with abdominal radiotherapy and abdominal surgery only. In addition, frequencies of pregnancy outcomes according to cancer type compared to comparison subjects were calculated.

Calendar time was categorized into two time periods (1991-2002 and 2003-2013), maternal age into four categories (under 25 years, 25-29 years, 30-34 years and 35 years or more) and gestational age as less than 32 weeks (early preterm), 32-36 weeks (preterm), 37-41 weeks (full term) or more than 42 weeks (post-term). Information on maternal smoking was available as smokers, nonsmokers and those with missing information.

To identify the reasons for preterm delivery we compared pregnancy related conditions among preterm (less than 37 gestational weeks) and term pregnancies (37-41 gestational weeks) using conditional logistic regression adjusting for maternal age at delivery, maternal smoking and early onset cancer. To study whether survivors with pregnancy related condition have an excess risk for preterm delivery compared to corresponding comparison subjects and survivors without pregnancy related condition, we included interactions and tested their significance using the likelihood ratio tests.

We conducted a systematic literature search in PubMed with combination of the terms "pregnancy outcomes", "preterm", "delivery", "cancer" and "survivorship" for studies on humans and published in English up to April 2018. Inclusion criteria for studies on pregnancies or deliveries in childhood, adolescent and young adult and adult female cancer survivors were carefully examined.

Analyses were conducted using STATA 14.0. 


\section{Ethics}

Our study protocol, including the use of administrative health data, was approved by the National Institute for Health and Welfare and Central Population Register (Dnro 918/410/14) and includes an ethical evaluation of the study (Dnro THL/1/5.05.00/2014). No identifiable personal-level data were available in the analysis and patients were not contacted.

\section{Results}

The descriptive characteristics of survivors and female comparison subjects are presented in Table 1 . The age at delivery was slightly higher among survivors than among population comparison (mean age 29.7 and 27.6 years respectively, $p<0.001$ ). Survivors tended to give birth more often during the most recent time period compared to comparison subjects $(p<0.001)$.

Both preterm delivery and low birth weight were more common among survivors than comparisons as $7.4 \%$ of cancer survivors compared to $5.2 \%$ of comparisons delivered preterm $(p=0.004$ and $p=0.002)$.

The proportion of preterm deliveries among survivors remained stable (7.4\%) when comparing the earlier time period of 1991-2002 to the most recent available time period of 2003-2013.

The patient characteristics of the survivors are listed in Table 2. In all, $41.6 \%$ of the survivors delivered within 6 years of their cancer diagnosis. The largest diagnostic group consisted of carcinomas and other malignant epithelial neoplasms (42.2\%), followed by lymphomas (18.7\%), leukemia (10.3\%) and tumors of the central nervous system (10.3\%). In all, $40.6 \%$ of cancer patients had received only surgical treatment, while $31.9 \%$ had received radiotherapy and $31.7 \%$ had received chemotherapy according to treatment data from the first 4 months after diagnosis recorded in the FCR. When it comes to tumor behavior, $5.0 \%$ were benign, $2.4 \%$ semimalignant and $92.6 \%$ malignant.

During pregnancy, 394 (22.5\%) survivors compared to $906(17.7 \%)$ population comparisons were hospitalized (OR 1.45, 95\% CI 1.25-1.68). In most cases hospitalization was due to reasons other than vaginal bleeding, pre-eclampsia or threatened preterm labor (OR 1.46, 95\% CI 1.23-1.73) (Table 3).

When it comes to pregnancy related conditions possibly leading to medically induced preterm birth, we found that $18(1.8 \%)$ survivors compared to $20(0.9 \%)$ comparisons suffered from intrahepatic cholestasis (OR 2.86, 95\% CI 1.09-7.49) and $42(4.2 \%)$ survivors compared to $46(2.1 \%)$ comparisons were diagnosed with fear of childbirth (OR 2.25, 95\% CI 1.31-3.85). Also mental disorders and diseases of the nervous system were more common among survivors as $28(2.8 \%)$ survivors and $16(0.7 \%)$ comparison subjects suffered from these conditions (OR 5.89, 95\% CI 2.31-15.00). However, the overall risk for vaginal bleeding, pre-eclampsia and gestational diabetes was not increased among survivors compared to comparisons. It is noteworthy that survivors $(49.6 \%)$ more often than
Table 2. Diagnostic characteristics of female cancer survivors experiencing their first postdiagnosis pregnancy

\begin{tabular}{|c|c|c|}
\hline Characteristics & Subcategory & $\begin{array}{l}\text { Survivors } \\
(N=1,753) N \\
(\%)\end{array}$ \\
\hline \multirow{3}{*}{$\begin{array}{l}\text { Year of } \\
\text { diagnosis }\end{array}$} & $1957-1972$ & $12(0.68)$ \\
\hline & 1973-1992 & $666(37.99)$ \\
\hline & $1993-2012$ & $\begin{array}{l}1,075 \\
(61.32)\end{array}$ \\
\hline \multirow{3}{*}{$\begin{array}{l}\text { Age at } \\
\text { diagnosis }\end{array}$} & $0-14$ & $398(22.70)$ \\
\hline & $15-24$ & $770(43.92)$ \\
\hline & $25-39$ & $585(33.37)$ \\
\hline \multirow{3}{*}{$\begin{array}{l}\text { Time from } \\
\text { diagnosis to } \\
\text { delivery } \\
\text { (years) }\end{array}$} & $0-5$ & 729 (41.59) \\
\hline & $6-10$ & $474(27.04)$ \\
\hline & $11-38$ & $550(31.37)$ \\
\hline \multirow[t]{17}{*}{ Cancer type } & Leukemia & $181(10.33)$ \\
\hline & Lymphoma & $328(18.71)$ \\
\hline & Central Nervous System & $181(10.33)$ \\
\hline & Sympathetic Nervous System & $30(1.71)$ \\
\hline & Retinoblastoma & $12(0.68)$ \\
\hline & Renal Tumors & $38(2.17)$ \\
\hline & Malignant bone Tumors & $35(2.00)$ \\
\hline & Soft Tissue and other Sarcomas & $72(3.93)$ \\
\hline & $\begin{array}{l}\text { Germ cell, Gonadal and } \\
\text { Trophoblastic neoplasms }\end{array}$ & $70(3.99)$ \\
\hline & $\begin{array}{l}\text { Carcinomas and other malignant } \\
\text { epithelial neoplasms }\end{array}$ & $739(42.16)$ \\
\hline & Digestive system & $120(6.85)$ \\
\hline & Breast & $80(4.56)$ \\
\hline & Melanoma of the skin & $183(10.44)$ \\
\hline & Thyroid gland & $246(14.03)$ \\
\hline & Others & $110(6.27)$ \\
\hline & Others & $16(0.91)$ \\
\hline & Missing & $55(3.14)$ \\
\hline \multirow{4}{*}{$\begin{array}{l}\text { Cancer } \\
\text { treatment }\end{array}$} & Chemotherapy & $556(31.72)$ \\
\hline & Radiotherapy & $559(31.89)$ \\
\hline & Surgery, only & $712(40.62)$ \\
\hline & Missing & $219(12.49)$ \\
\hline
\end{tabular}

comparisons $(42.7 \%)$ had undergone a 2 -h oral glucose tolerance test $\left(x^{2}\right.$-test, $\left.p<0.001\right)$.

In our data, women (both survivors and comparisons) who delivered preterm more often had hospital stays during pregnancy and more often stayed at least 7 days in the hospital after birth compared to women who delivered full term. Women delivering preterm also had an increased risk for pre- 
Table 3. Adjusted odd ratios (ORs) for pregnancy outcomes between 1991 and 2013 among female cancer survivors with their first postdiagnosis pregnancy compared to first pregnancies in a matched female comparison group

\begin{tabular}{|c|c|c|c|}
\hline Pregnancy outcome & $\begin{array}{l}\text { Survivors } \\
(N=1,753)(\%)\end{array}$ & $\begin{array}{l}\text { Comparisons } \\
(N=5,123)(\%)\end{array}$ & $\begin{array}{l}\text { Adjusted } \mathrm{OR}^{1} \\
(95 \% \mathrm{Cl})\end{array}$ \\
\hline Hospital stay $>7$ days & $190(10.84)$ & $446(8.71)$ & $1.10(0.90-1.34)$ \\
\hline Any hospitalization & $394(22.48)$ & $906(17.68)$ & $1.45(1.25-1.68)$ \\
\hline $\begin{array}{l}\text { Hospitalization for other reason than vaginal bleeding, } \\
\text { pre-eclampsia or threatened preterm labor }\end{array}$ & $280(15.97)$ & $617(12.04)$ & $1.46(1.23-1.73)$ \\
\hline \multicolumn{4}{|l|}{$\begin{array}{l}\text { Pregnancy outcomes possibly leading to } \\
\text { spontaneous preterm delivery }\end{array}$} \\
\hline Hospitalization for threatened preterm labor & $49(2.80)$ & $105(2.05)$ & $1.39(0.91-2.12)$ \\
\hline Preterm rupture of the membranes ${ }^{2}$ & $41(4.08)$ & $68(3.04)$ & $1.21(0.67-2.17)$ \\
\hline \multicolumn{4}{|l|}{$\begin{array}{l}\text { Pregnancy outcomes possibly leading to } \\
\text { medically iatrogenic preterm birth }\end{array}$} \\
\hline Hospitalization for vaginal bleeding & $24(1.37)$ & $52(1.02)$ & $1.31(0.74-2.31)$ \\
\hline Hospitalization for pre-eclampsia & $97(5.53)$ & $258(5.04)$ & $1.11(0.85-1.45)$ \\
\hline Gestational diabetes $^{2}$ & $109(10.85)$ & $196(8.77)$ & $1.06(0.78-1.43)$ \\
\hline Intrahepatic cholestasis ${ }^{2}$ & $18(1.79)$ & $20(0.89)$ & $2.86(1.09-7.49)$ \\
\hline Fear of childbirth ${ }^{2}$ & $42(4.18)$ & $46(2.06)$ & $2.25(1.31-3.85)$ \\
\hline Mental disorders and diseases of the nervous system ${ }^{2}$ & $28(2.79)$ & $16(0.72)$ & $5.89(2.31-15.00)$ \\
\hline
\end{tabular}

$\mathrm{OR}$, odd ratio; $\mathrm{Cl}$, confidence interval. Bold indicates statistically significant odds ratios. $p$-value $<0.05$.

${ }^{1}$ Adjusted for maternal age, gestational age and maternal smoking.

${ }^{2}$ Data available from 2004 to 2013 (1,005 survivors and 2,236 female controls).

eclampsia and vaginal bleeding (Adjusted OR for preterm labor ${ }^{1}$ in Table 4).

Analyzing the effect that cancer alone had on preterm delivery (Adjusted OR for early onset cancer ${ }^{2}$ in Table 4), we found that survivors did not have an excess risk for preterm delivery compared to comparison subjects with the same condition in any of the outcomes. However, we identified a statistically significant increased risk for preterm delivery among cancer survivors with vaginal bleeding (OR 1.35, 95\% CI 1.07-1.71) and pre-eclampsia (1.35, 95\% CI 1.06-1.72) compared to comparison subjects with the same condition.

Looking at sub-analyses, stratifying by time from cancer diagnosis to delivery (Supporting Information Table S1), survivors delivering within 6 years of cancer treatment had the

Table 4. Adjusted odd ratios for pregnancy outcomes between 1991 and 2013 comparing preterm deliveries (gestational age below 37 weeks) with full-term deliveries as well as adjusted odd ratios for pregnancy outcomes due to early onset cancer

\begin{tabular}{|c|c|c|c|c|}
\hline Pregnancy outcome & $\begin{array}{l}\text { Preterm } \\
(N=397)(\%)\end{array}$ & $\begin{array}{l}\text { Full term } \\
(N=6,466)(\%)\end{array}$ & $\begin{array}{l}\text { Adjusted OR for } \\
\text { preterm labor }^{1} \\
(95 \% \mathrm{Cl})\end{array}$ & $\begin{array}{l}\text { Adjusted OR for } \\
\text { early onset cancer } 2 \\
(95 \% \mathrm{Cl})\end{array}$ \\
\hline Hospital stay $>7$ days & $151(38.04)$ & $484(7.49)$ & $9.14(6.47-12.91)$ & $1.06(0.77-1.46)$ \\
\hline Any hospitalization & $161(40.55)$ & $1,137(17.58)$ & $3.33(2.52-4.39)$ & $1.14(0.84-1.55)$ \\
\hline $\begin{array}{l}\text { Hospitalization for other reason than } \\
\text { vaginal bleeding, pre-eclampsia } \\
\text { or threatened preterm labor }\end{array}$ & $94(23.68)$ & $801(12.39)$ & $2.11(1.56-2.87)$ & $1.38(1.06-1.80)$ \\
\hline \multicolumn{5}{|c|}{ Pregnancy outcomes possibly leading to spontaneous preterm delivery } \\
\hline Hospitalization for threatened preterm labor & $47(11.84)$ & $107(1.65)$ & $8.48(4.65-15.47)$ & $1.21(0.94-1.55)$ \\
\hline Preterm rupture of the membranes ${ }^{3}$ & $39(19.70)$ & $70(2.30)$ & $14.56(5.32-39.89)$ & $1.25(0.83-1.89)$ \\
\hline \multicolumn{5}{|l|}{$\begin{array}{l}\text { Pregnancy outcomes possibly leading to } \\
\text { medically iatrogenic preterm birth }\end{array}$} \\
\hline Hospitalization for vaginal bleeding & $12(3.02)$ & $64(0.99)$ & $5.70(2.11-15.40)$ & $1.35(1.07-1.71)$ \\
\hline Hospitalization for pre-eclampsia & $51(12.85)$ & $309(4.72)$ & $3.02(1.96-4.65)$ & $1.35(1.06-1.72)$ \\
\hline Gestational diabetes $^{2}$ & $19(9.60)$ & $286(9.41)$ & $0.87(0.42-1.80)$ & $1.44(0.98-2.10)$ \\
\hline Intrahepatic cholestasis ${ }^{2}$ & $4(2.02)$ & $34(1.12)$ & $1.92(0.33-11.10)$ & $1.35(0.94-1.95)$ \\
\hline Fear of childbirth ${ }^{2}$ & $1(0.51)$ & $87(2.86)$ & $0.18(0.02-1.45)$ & $1.39(0.96-2.01)$ \\
\hline Mental disorders and diseases of the nervous system ${ }^{2}$ & $4(2.02)$ & $40(1.32)$ & $1.99(0.38-10.46)$ & $1.40(0.97-2.02)$ \\
\hline
\end{tabular}

$\mathrm{OR}$, odd ratio; $\mathrm{Cl}$, confidence interval. Bold indicates statistically significant odds ratios.

${ }^{1}$ Adjusted for maternal age, maternal smoking and early onset cancer.

${ }^{2}$ Adjusted for maternal age and maternal smoking.

${ }^{3}$ Data available from 2004-2013 (198 preterm and 3,040 full term deliveries) 
highest risk for overall hospitalization (OR 1.75, 95\% CI 1.34-2.30) and hospitalization for other reasons than vaginal bleeding, pre-eclampsia or threatened preterm labor (OR 1.76, 95\% CI 1.30-2.39). According to diagnostic age (Supporting Information Table 2), adolescents and young adults (age 15-25 years) had the highest risk for overall hospitalization (OR 1.62, 95\% CI 1.24-2.10), hospitalization due to vaginal bleeding (OR 2.83, 95\% CI 1.11-7.18) and fear of childbirth (OR 3.72, 95\% CI 1.15-12.02) compared to comparison subjects. Regarding cancer treatments, survivors treated with surgery alone had the highest risk for overall hospitalization (OR 1.58, 95\% CI 1.16-2.14) but the risk was also increased among survivors treated with chemotherapy and radiation. Survivors treated with surgery alone was the only treatment group with increased risk for fear of childbirth (OR 3.67, 95\% CI 1.15-11.74) compared to comparisons (Supporting Information Table 3). Supplementary analyses of survivors treated with abdominal radiotherapy (107 survivors) and abdominal surgery only (164 survivors) did not show an increased risk for pregnancy related conditions but the numbers were small.

The risk for threatened preterm labor, preterm rupture of membranes, pre-eclampsia or gestational diabetes was not increased in any of the subgroups.

Frequencies of pregnancy outcomes according to the most common cancer types can be found in Supporting Information tables (Supporting Information Tables 4 and 5).

\section{Discussion}

We studied different pregnancy related conditions that could explain the increased risk for preterm delivery observed among early onset cancer survivors.

In our study, cancer survivors had a significantly increased risk for preterm delivery compared to comparison subjects. An overall increased risk for hospitalization during pregnancy, intrahepatic cholestasis, fear of childbirth and mental disorders and diseases of the nervous system complicating pregnancy and labor was also observed. We found a statistically significant increased risk for preterm delivery among cancer survivors with vaginal bleeding and pre-eclampsia compared to comparison subjects with the same condition.

The main strengths of our study is population-based data originating from consistent, homogenous and reliable national registers covering a large number of pregnancies over a long period of time. ${ }^{17-19}$ Registry based data provided information on outcomes studied as well as important confounders, making it possible to adjust for different factors that might interfere with the outcomes. Registry data are, as opposed to self-reported data, free of recall and reporting bias because practically all individuals can be followed for the studied outcomes. Based on a systematic literature search, our study is the first to explore preterm delivery among cancer survivors and matched comparison subjects according to different pregnancy related conditions.
The main limitation of our study is lack of treatment exposure data. As the outcomes studied, are affected by local damage to the reproductive system, these exposures are particularly important. Furthermore, data did not contain complete information on all reasons for hospitalization during pregnancy and some diagnostic groups were unspecific or combined several different diseases, mental disorders and diseases of the nervous system as an example. Information on socioeconomic status was missing in one third of the study population because young women are often students or at home taking care of their children. Instead, we used data on smoking as a proxy when adjusting for socioeconomic factors, since this has been found to be a good proxy for socioeconomic status in Finland. ${ }^{20}$ Subgroup analyses by cancer type resulted in such small numbers, that reporting risks was not sensible.

As in previous studies, ${ }^{6-13}$ we found survivors to have a significantly increased risk for preterm delivery compared to comparison subjects. We also found a $45 \%$ higher risk for overall hospitalization among survivors compared to population comparisons but could not identify any specific reasons for the increased risk for hospitalization as most survivors belonged to the category hospitalization for other reasons than vaginal bleeding, pre-eclampsia or threatened preterm labor. One recent study found that childhood cancer survivors in Finland have a relative risk of 1.87 compared to matched controls to be hospitalized for all medical indications in adult life, $^{21}$ thus cancer survivors suffer from late morbidities, namely chronic diseases, after cancer.

An Australian study ${ }^{8}$ reported an increased risk for preeclampsia (OR 1.44, 95\% CI 1.13-1.87), gestational diabetes (OR 1.38, 95\% CI 1.09-2.98) and hospital stays exceeding 5 days (OR 3.01, 95\% CI 1.72-5.58) among cancer survivors compared to women without a history of cancer, which is in conflict with our results. However, the risks for vaginal bleeding during pregnancy, preterm rupture of the amniotic membranes and threatened preterm labor were not increased. our study covered a large number of first completed pregnancies over a long period of time. As a comparison group they included women without a history of cancer, giving birth during the same period of time and matched on maternal age group, parity and year of delivery. In our study, only cancer survivors diagnosed as adolescents, young adults and adults (15-39 years) were included.

Another recent study ${ }^{22}$ found that treatment with abdominal radiotherapy increases the risk of hypertension complicating pregnancy in Wilms tumor survivors as well as gestational diabetes and anemia in all survivors. our study covered only childhood cancer survivors (diagnosed at the age of 0-14 years) and included also multiparous women.

According to our study, survivors had a more than twofold increased risk for fear of childbirth, an almost three-fold increased risk for intrahepatic cholestasis and close to six-fold increased risk for the combined group of mental disorders and diseases of the nervous system complicating pregnancy 
and childbirth, which to our knowledge are new findings. Studies mostly conducted in the Nordic countries, have found that $6-10 \%$ of all pregnant women suffer from severe fear of childbirth. ${ }^{23,24}$ Fear of childbirth is often the underlying reason for the mothers' request for cesarean section and could, if untreated, lead to unnecessary cesarean section without medical indication. Mental health problems are twice as common among women with fear of childbirth as in nonfearful controls. ${ }^{25}$ Depression is the most common mental disease among pregnant women with a prevalence of $10-15 \%$ of all pregnant women. ${ }^{26}$ According to one study, cancer survivors in the United States reported medication use for anxiety and depression at rates nearly two times those reported by the general public. ${ }^{27}$ Anxiety and depression are both factors that have been associated with an increased risk for both iatrogenic and spontaneous preterm delivery. ${ }^{28}$ Intrahepatic cholestasis is associated with an increased risk for fetal distress and preterm labor. ${ }^{29}$

We found a statistically significant increased risk for preterm deliveries among cancer survivors with vaginal bleeding and preeclampsia compared to comparison subjects with the same condition. Our results suggest that these pregnancy-related conditions, possibly leading to medically induced preterm birth, tend to be more severe among survivors compared to comparison subjects. Another possibility is that the history of cancer influences decision making, lowering the threshold for medical intervention in these survivors, leading to preterm delivery.

Early onset cancer survivors have an increased risk for preterm deliveries compared to population comparison subjects. This could be explained either by certain pregnancy-related conditions which seem to be more severe among survivors than comparison subjects, possibly leading to medically induced preterm birth or the history of cancer, lowering the threshold for medical interventions leading to preterm delivery among survivors. Survivors also have an increased risk for hospitalization during pregnancy, intrahepatic cholestasis, fear of childbirth and mental disorders and diseases of the nervous system compared to female comparisons. Health professionals treating these women should be aware of these risks and consider individualized follow-up when indicated. In general, however, our results are reassuring when it comes to pregnancies among cancer survivors.

\section{Acknowledgements}

This study was supported by a grant from the Cancer Society of Finland and by a grant from the Gyllenberg Foundation.

\section{References}

1. Madanat-Harjuoja LM, Pokhrel A, Kivivuori SM, et al. Childhood cancer survival in Finland (1953-2010): a nation-wide population-based study. Int J Cancer 2014;135:2129-34.

2. Gatta G, Botta L, Rossi S, et al. Childhood cancer survival in Europe 1999-2007: results of EUROCARE-5-a population based study. Lancet Oncol 2014;15:35-47.

3. Barton S, Najita J, Ginsburg E, et al. Infertility, infertility treatment, and achievement of pregnancy in female survivors of childhood cancer: a report from the childhood cancer survivor study cohort. Lancet Oncol 2013;14:873-81.

4. Meacham L, Chow E, Ness K, et al. Cardiovascular risk factors in adult survivors of pediatric cancer-a report from the childhood cancer survivor study. Cancer Epidemiol Biomarkers Prev 2010;19:170-81.

5. Holmqvist A, Olsen J, Andersen K, et al. Adult life after childhood cancer in Scandinavia: diabetes mellitus following treatment for cancer in childhood. Eur J Cancer 2014;50:1169-75.

6. Melin J, Heinävaara S, Malila N, et al. Adverse obstetric outcomes among early onset cancer survivors in Finland. Obstet Gynecol 2015;126: 803-10.

7. Clark H, Kurinczuk JJ, Lee AJ, et al. Obstetric outcomes in cancer survivors. Obstet Gynecol 2007; 110:849-54.

8. Haggar FA, Pereira G, Preen D, et al. Adverse obstetric and perinatal outcomes following treatment of adolescent and young adult cancer: a populationbased cohort study. PLoS One 2014;9:e113292.

9. Madanat-Harjuoja LM, Malila N, Lähteenmäki PM, et al. Preterm delivery among female survivors of childhood, adolescent and young adulthood cancer. Int J Cancer 2010;127:1669-79.
10. Green DM, Whitton JA, Stovall M, et al. Pregnancy outcome of female survivors of childhood cancer: a report from the childhood cancer survivor study. Am J Obstet Gynecol 2002; 187:1070-80.

11. Anderson C, Engel S, Mersereau J, et al. Birth outcomes among adolescent and young adult cancer survivors. JAMA Oncol 2017;3:1078-84.

12. Arbyn M, Kyrgiou M, Simoens C, et al. Perinatal mortality and other severe adverse pregnancy outcomes associated with treatment of cervical intraepithelial neoplasia: meta-analysis. BMJ 2008; 337:a1284.

13. Hartnett KP, Ward KC, Kramer MR, et al. The risk of preterm birth and growth restriction in pregnancy after cancer. Int J Cancer 2017;141: 2187-96.

14. Thomson F, Shanbhag S, Templeton A, et al. Obstetric outcome in women with subfertility. BJOG 2005;112:632-7.

15. Ananth C, Vintzileos A. Maternal-fetal conditions necessitating a medical intervention resulting in preterm birth. AJOG 2006;195:1557-63.

16. Green DM, Lange JM, Peabody EM, et al. Pregnancy outcome after treatment for Wilms tumor: a report from the national Wilms tumor long-term follow-up study. J Clin Oncol 2010;28:2824-30.

17. Leinonen MK, Miettinen J, Heikkinen S, et al. Quality measures of the population-based Finnish cancer registry indicate sound data quality for solid malignant tumours. Eur J Cancer 2017;77:31-9.

18. Steliarova-Foucher E, Stiller C, Lacour B, et al. International classification of childhood cancer, third edition. Cancer 2005;103:1457-67.

19. Gissler M, Shelley J. Quality of data on subsequent events in a routine medical birth register. Med Inform Internet Med 2002;27:33-8.
20. Jaakkola N, Jaakkola S, Gissler M, et al. Smoking during pregnancy in Finland: determinants and trends 1987-1997. Am J Public Health 2001;91: 284-6.

21. de Fine LS, Rugbjerg K, Gudmundsdottir T, et al. Long-term disease burden in the adult life after childhood cancer in Scandinavia (ALiCCS) study: a cohort study of 21,297 childhood cancer survivors. PLoS Med 2017;14:e1002296.

22. Reulen RC, Bright CJ, Winter DL, et al. Pregnancy and labor complications in female survivors of childhood cancer: the British childhood cancer survivor study. J Natl Cancer Inst 2017;109:djx056. https://doi.org/10.1093/jnci/djx056.

23. Saisto T, Ylikorkala O, Halmesmäki E. Factor associated with a fear of childbirth in second pregnancies. Obstet Gynecol 1999;94:679-82.

24. Melender HL. Experiences of fear associated with pregnancy and childbirth. Birth 2002;29:101-11.

25. Rouhe H, Salmela-Aro K, Gissler M, et al. Mental health problems common in women with fear of childbirth. BJOG 2011;118:1104-11.

26. Gavin NI, Gaynes BN, Lohr KN, et al. Perinatal depression: a systematic review of prevalence and incidence. Obstet Gynecol 2005;106:1071-83.

27. Hawkins NA, Soman A, Buchanan Lunsford N, et al. Use of medications for treating anxiety and depression in cancer survivors in the United States. J Clin Oncol 2017;35:78-85.

28. Tanne JH. Women with severe depressive symptoms are twice as likely to have preterm delivery. BMJ (Clinical research ed). 2008;337:a2312.

29. Geenes V, Chappell L, Seed P, et al. Association of severe intrahepatic cholestasis of pregnancy with adverse pregnancy outcomes: a prospective population-based case-control study. Hepatology 2014;59:1482-91. 